\title{
Single-Level Minimally Invasive Transforaminal Lumbar Interbody Fusion Provides Sustained Improvements in Clinical and Radiological Outcomes up to 5 Years Postoperatively in Patients with Neurogenic Symptoms Secondary to Spondylolisthesis
}

\author{
Hamid Rahmatullah Bin Abd Razak ${ }^{1}$, Priyesh Dhoke ${ }^{2}$, Kae-Sian Tay ${ }^{1}$, William Yeo ${ }^{1}$, Wai-Mun Yue ${ }^{1}$ \\ ${ }^{1}$ Department of Orthopaedic Surgery, Singapore General Hospital, Singapore \\ ${ }^{2}$ Shanta Spine Institute, Nagpur, India
}

Study Design: Retrospective review of prospective registry data.

Purpose: To determine 5-year clinical and radiological outcomes of single-level instrumented minimally invasive transforaminal lumbar interbody fusion (MIS-TLIF) in patients with neurogenic symptoms secondary to spondylolisthesis.

Overview of Literature: MIS-TLIF and open approaches have been shown to yield comparable outcomes. This is the first study to assess MIS-TLIF outcomes using the minimal clinically important difference (MCID) criterion.

Methods: The outcomes of 56 patients treated by a single surgeon, including the Oswestry disability index (ODI), neurogenic symptom score, short-form 36 questionnaire (SF-36), and visual analog scale (VAS) scores for back pain (BP), and leg pain (LP), were collected prospectively for up to 5 years postoperatively. Radiological outcomes included adjacent segment degeneration, fusion, cage subsidence, and screw loosening rates.

Results: Our patients were predominantly female $(71.4 \%)$ and had a mean age of $53.7 \pm 11.3$ years and mean body mass index of $25.7 \pm 3.7 \mathrm{~kg} / \mathrm{m}^{2}$. The mean operative time, blood loss, time to ambulation, and hospitalization were $167 \pm 49$ minutes, $126 \pm 107 \mathrm{~mL}$, $1.2 \pm 0.4$ days, and $2.8 \pm 1.1$ days, respectively. The mean fluoroscopic time was $58.4 \pm 33$ seconds, and the mean postoperative intravenous morphine dose was $8 \pm 2 \mathrm{mg}$. Regarding outcomes, postoperative scores improved relative to preoperative scores, and this was sustained across various time points for up to 5 years $(p<0.001)$. Improvements in ODI, SF-36, VAS-BP, and VAS-LP all met the MCID criterion. Notably, $5.4 \%$ of our patients developed clinically significant adjacent segment disease during follow-up, and 7 minor complications were reported.

Conclusions: Single-level instrumented MIS-TLIF is suitable for patients with neurogenic symptoms secondary to lumbar spondylolisthesis and is associated with an acceptable complication rate. Both clinical and radiological outcomes were sustained up to 5 years postoperatively, with many patients achieving an MCID.

Keywords: Lumbar spine; Spondylolisthesis, minimally invasive surgery; Transforaminal lumbar interbody fusion; Minimal clinically important difference

Received Jun 6, 2016; Revised Sep 11, 2016; Accepted Sep 15, 2016

Corresponding author: Wai-Mun Yue

Department of Orthopaedic Surgery, Singapore General Hospital, Outram Road, 640633 Singapore

Tel: +65-63214603, Fax: +65-62248100, E-mail: yuewm@singnet.com.sg 


\section{Introduction}

Instrumented lumbar interbody fusion is a common procedure used to treat various pathologies of the lumbar spine, including spondylolisthesis. The goal of fusion is to achieve stable fusion of spinal segments with good vertebral height and alignment [1]. Surgical approaches for fusion include posterior lumbar interbody fusion (PLIF) [2], transforaminal lumbar interbody fusion (TLIF) [3], or extreme lateral interbody fusion [4], each having its own benefits and drawbacks. TLIF, which avoids thecal sac retraction and reduces trauma to the lumbar musculature, facet joints, and lamina, has increased in popularity relative to PLIF [5]. Lumbar fusion can be performed via open or minimally invasive surgery (MIS). Open TLIF has been proven safe for achieving spinal fusion and restoring both disc height and spinal alignment in patients with symptomatic spondylolisthesis and degenerative disc disease in the lumbar spine $[1,6]$. However, open TLIF is associated with significant postoperative morbidity due to the deep, extensive soft tissue dissection required for surgical exposure, and inadvertent damage to the lumbar spine musculature may cause significant postoperative pain and delayed rehabilitation [7]. MIS-TLIF has been shown to be comparable to open TLIF regarding midterm clinical outcomes and fusion rates, and to confer the additional benefits of reduced postoperative pain and intraoperative blood loss, earlier rehabilitation, and shorter hospitalization [8-10].

Although several studies have reported the outcomes of MIS-TLIF, only one has reported 5-year clinical and radiological outcomes for single-level MIS-TLIF in patients with neurogenic symptoms secondary to lumbar spondylolisthesis [11]. Therefore, to the best of our knowledge, this is the first study to assess clinical outcomes using the minimal clinically important difference (MCID) criterion. In this study, we sought to determine whether patients with neurogenic symptoms secondary to lumbar spondylolisthesis who underwent instrumented single-level MIS-TLIF would achieve sustained improvements in both clinical and radiological outcomes for up to 5 years postoperatively.

\section{Materials and Methods}

After obtaining Institutional Review Board approval, we conducted a retrospective cohort analysis of 56 con- secutive patients with prospectively collected data from the hospital's spine registry. Patients who underwent MIS-TLIF performed by the senior author of this paper (W.M.Y.) between January 2004 and March 2009 and who had completed 5 years of follow-up were included in our study. The inclusion criteria were as follows: patients with (1) symptomatic lumbar spondylolisthesis with neurogenic pain; (2) a history of single-level MIS-TLIF; (3) neurogenic pain and/or any of the following: (a) low back pain (BP), (b) unilateral or bilateral impaired sensation over a lower limb dermatome, (c) unilateral or bilateral impaired power over a lower limb myotome; and (4) complete data from 5 years of follow-up assessments. The exclusion criteria were (1) previous spinal instrumentation, (2) thoracolumbar spinal instrumentation, (3) spinal infection, (4) tumor-related spinal pathology, and (5) acute spinal trauma. All patients underwent a preoperative assessment involving a detailed neurological examination and radiological imaging, including static (anteroposterior and lateral) and dynamic (flexion and extension) plain lumbar spine radiography and magnetic resonance imaging (MRI). Surgery was offered to patients who had received conservative treatment (physiotherapy, oral analgesia, and activity modification) for at least 6 months without significant symptom relief.

The details of the operative MIS-TLIF techniques were described by the senior author in a previous publication [8]. The TLIF surgical approach was decided based on the symptomatic side; if both sides were symptomatic, the incision was made on the side involving more severe pathology. A mobile C-arm X-ray machine was used to confirm the desired operative level. A parasagittal incision was made 3-5 $\mathrm{cm}$ lateral to the midline, and sequential soft tissue dilators were inserted down to the facet complex, after which facetectomy was performed using a high-speed burr from the lateral to the medial direction to expose the posterior lateral disc aspect. Next, a discectomy was performed and the endplates were prepared. After performing disc space distraction using intradiscal spreaders, a bone graft was placed anteriorly and contralaterally to the annulotomy, followed by an interbody cage. Fluoroscopy was used to ensure satisfactory placement of the interbody cage. Next, decompression was achieved via removal of the remaining ipsilateral facet and lamina and resection of the lateral margin of the ligamentum flavum to expose the ipsilateral exiting and traversing roots. In cases with bilateral disease indicated by symptoms and MRI findings, the 
tubular retractor was angled medially and the patient was tilted to visualize the contralateral side and allow decompression where indicated. After adequate decompression, a percutaneous pedicle screw-rod construct was placed through the same incision and a similar construct was inserted through a contralateral incision. Compression was applied to restore lordosis before finally tightening the construct. Hemostasis and wound irrigation were performed before layered closure. Fifteen patients received autogenous posterior iliac crest bone grafts through separate incisions, and 41 patients received local lamina bone grafts with the demineralized bone matrix (DBM) Osteofil (Medtronic, Minneapolis, MN, USA); none received bone morphogenetic protein. Comorbidities and intraoperative blood losses were determined from anesthetic charts.

Postoperatively, patients were treated via a standardized clinical protocol. All patients sat up in bed postoperatively and were encouraged to ambulate from the first postoperative day; those who could ambulate independently, with or without an aid, were considered independently ambulant. Patients were deemed fit for discharge to their homes after they successfully ambulated $20-30 \mathrm{~m}$ independently and were found to be competent with stair climbing, if required. The hospitalization stay duration was the number of days required to achieve sufficient independence to be discharged home. All patients were given patient-controlled analgesia (morphine) for postoperative pain relief. After discharge from hospital, the patients followed up with the surgeon at 2 and 6 weeks, 3 and 6 months, 1 year, and annually thereafter. Plain lumbar spine radiographs were obtained at each follow-up appointment to assess fusion and monitor complications. If indicated, MRI or computed tomography (CT) scans were ordered for further assessment and confirmation of complications. Complications were categorized as clinical, including new or worsening neurological deficit(s) and wound infections, or technical, including screw loosening and cage subsidence.

Two independent assessors who were not directly involved in patient care collected all patients' demographic information and functional scores at our institutional Orthopedic Diagnostic Centre. The assessed functional outcomes included the Oswestry disability index (ODI), neurogenic symptoms score (NSS), 36-item short form health survey (SF-36), and visual analogue scale (VAS) scores for BP and leg pain (LP) [12-15]. These scores were collected prospectively before surgery and at 6 months, 2 years, and 5 years postoperatively. For the ODI, NSS, and VAS, lower scores indicated better outcomes. Two independent assessors evaluated fusion rates using the Bridwell classification (Table 1) and adjacent segment degeneration (ASDeg) using the University of California at Los Angeles (UCLA) Grading Scale for Intervertebral Space Degeneration (Table 2) at 6 months, 2 years, and 5 years postoperatively $[16,17]$. Note that ASDeg should be distinguished from clinically significant adjacent segment disease (ASD). For our study purposes, ASDeg was defined as asymptomatic radiographic deterioration of segments adjacent to lumbar arthrodesis [18] and ASD was defined as the presentation of new symptoms referable to an adjacent level following successful MIS-TLIF at an index level [18]. The latter would not classically include

Table 1. Bridwell interbody fusion grading system

\begin{tabular}{ll} 
Grade & \multicolumn{1}{c}{ Description } \\
I & Fused with remodelling and trabeculae present \\
III & $\begin{array}{l}\text { Graft intact, not fully remodelled and incorporated, } \\
\text { but no lucency present }\end{array}$ \\
IV & $\begin{array}{l}\text { Graft intact, potential lucency present at top and } \\
\text { bottom of graft }\end{array}$ \\
\hline
\end{tabular}

Table 2. UCLA grading scale for adjacent segment degeneration

\begin{tabular}{lcrc} 
Grade & Disc-space narrowing & Osteophytes & End plate sclerosis \\
I & - & - & - \\
II & + & - & - \\
III & \pm & + & - \\
IV & \pm & \pm & + \\
\hline
\end{tabular}

The grade is based on the most severe radiographic finding evident on plain radiographs.

Patients were rated based on the worst category satisfied.

UCLA, University of California at Los Angeles; +, present; -, absent; \pm , either present or absent. 
sequelae to index arthrodesis such as axial pain, muscle spasm, or numbness. Radiographs were also assessed for screw loosening and cage subsidence at various time points. The MCID criterion was used to assess improvements in outcomes at various time points. We used MCID values of 13 for the ODI, 5 for the SF-36, 2 for the VAS$\mathrm{BP}$, and 3 for the VAS-LP $[19,20]$.

All statistical analyses were performed using the SPSS ver. 20 (IBM Corp., Armonk, NY, USA). We subjected 53 patients to an analysis of variance (ANOVA) with three pairwise comparisons. This sample size was intended to achieve $80 \%$ power with an $\alpha=0.05$. A repeated-measures ANOVA was used to compare clinical outcomes at various time points. Marascuilo's post hoc multiple proportion comparison was used to compare radiological outcomes at various time points. In all analyses, significance was

Table 3. Demographic and perioperative data of patients

\begin{tabular}{|c|c|}
\hline Variable & $\begin{array}{c}\text { Patients } \\
\text { undergoing } \\
\text { single-level } \\
\text { MIS-TLIF }(n=56)\end{array}$ \\
\hline \multicolumn{2}{|l|}{ Demographic data } \\
\hline Female & $40(71.4)$ \\
\hline Age (yr) & $53.7 \pm 11.3$ \\
\hline Body mass index $\left(\mathrm{kg} / \mathrm{m}^{2}\right)$ & $25.7 \pm 3.7$ \\
\hline \multicolumn{2}{|l|}{$\begin{array}{l}\text { No. of major co-morbidities (ischaemic } \\
\text { heart disease, hypertension, diabetes } \\
\text { mellitus) }\end{array}$} \\
\hline 1 Comorbidity & $22(39.2)$ \\
\hline 2 Comorbidities & $4(7.1)$ \\
\hline 3 Comorbidities & $1(1.8)$ \\
\hline \multicolumn{2}{|l|}{ Perioperative data } \\
\hline \multicolumn{2}{|l|}{ Spinal level fused } \\
\hline $\mathrm{L} 3 / \mathrm{L} 4$ & $6(10.7)$ \\
\hline L4/L5 & $39(69.6)$ \\
\hline L5/S1 & $11(19.7)$ \\
\hline Fluoroscopic team (sec) & $58.4 \pm 33$ \\
\hline Blood loss (mL) & $126 \pm 107$ \\
\hline Operative time (min) & $167 \pm 49$ \\
\hline Time to ambulate (day) & $1.24 \pm 0.4$ \\
\hline Length of stay (day) & $2.8 \pm 11$ \\
\hline Dose of intravenous PCA morphine (mg) & $8 \pm 2$ \\
\hline
\end{tabular}

Values are presented as number (\%) or mean \pm standard deviation. MIS-TLIF, minimally invasive transforaminal lumbar interbody fusion; PCA, patient-controlled analgesia. defined as $p<0.05$.

\section{Results}

Fifty-six patients followed up for a mean of $7.4 \pm 2.8$ years met the inclusion criteria and were analyzed. Demographic and preoperative data are presented in Table 3. The average patient who underwent MIS-TLIF was a relatively healthy middle-aged woman (mean age, 53.7 years; body mass index, $25.7 \mathrm{~kg} / \mathrm{m}^{2}$ ) with neurogenic symptoms secondary to L4/L5 spondylolisthesis.

The patients' clinical and radiological outcomes are summarized in Table 4. Early postoperative results at 1 and 3 months indicated good BP and LP relief, with mean VAS-BP and VAS-LP scores of $3 \pm 3$ at both time points. All patients exhibited improvements in all clinical outcomes by 6 months postoperatively relative to preoperative scores, and these improvements were sustained up to 5 years of follow-up. Changes in the outcome scores from the preoperative analysis to various follow-up time points were also significant. The MCIDs for mean ODI, SF-36, VAS-BP, and VAS-LP values were achieved at the 6-month follow-up and sustained until the 5-year followup. The proportions of patients achieving the MCID for each clinical outcome are illustrated in Table 5. Outcome trends over time are shown in Fig. 1.

Regarding bony fusion, $39.3 \%, 92.9 \%$, and $96.4 \%$ of our patients achieved grade 1 fusion by 6 months, 2 years, and 5 years, respectively. ASDeg was observed in $1.8 \%$, $19.7 \%$, and $39.3 \%$ of patients at the 6 -month, 2 -year, and

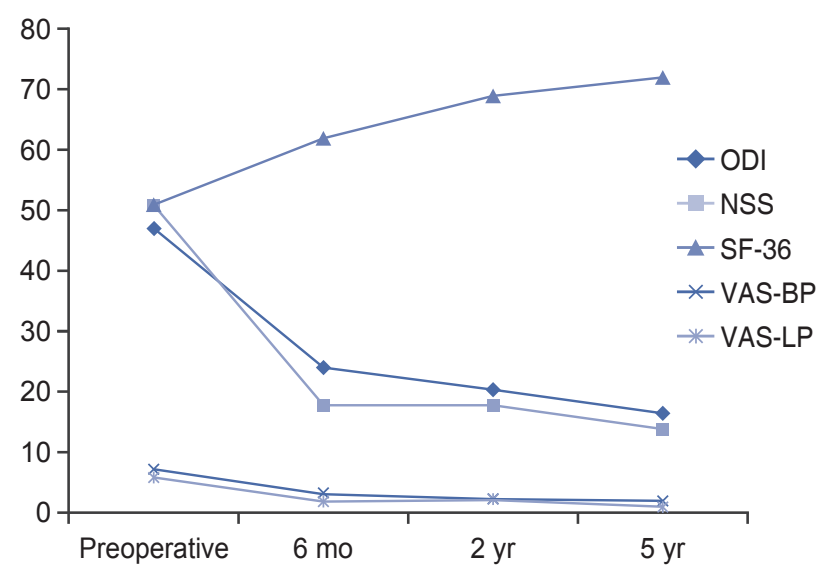

Fig. 1. Time trends of outcomes after single-level minimally invasive transforaminal lumbar interbody fusion. ODI, Oswestry disability index; NSS, neurogenic symptom score; SF-36, short-form 36 score; VAS, visual analogue scale score; BP, back pain; LP, leg pain. 
Table 4. Clinical and radiological outcomes

\begin{tabular}{|c|c|c|c|c|c|c|}
\hline Clinical outcomes & $\begin{array}{l}\text { Preoperative } \\
\qquad(\mathrm{n}=56)\end{array}$ & $\begin{array}{c}6 \text { mo } \\
\text { postoperative } \\
(n=56)\end{array}$ & $\begin{array}{c}2 \mathrm{yr} \\
\text { postoperative } \\
(\mathrm{n}=56)\end{array}$ & $\begin{array}{c}5 \mathrm{yr} \\
\text { postoperative } \\
(\mathrm{n}=56)\end{array}$ & MCID & $p$-value \\
\hline Oswestry disability index & $47 \pm 20$ & $24 \pm 19$ & $20 \pm 21$ & $16 \pm 19$ & 13 & $<0.0001$ \\
\hline Neurogenic symptom score & $51 \pm 30$ & $18 \pm 25$ & $18 \pm 26$ & $14 \pm 27$ & - & $<0.0001$ \\
\hline Short-form 36 score & $51 \pm 20$ & $62 \pm 23$ & $69 \pm 23$ & $72 \pm 20$ & 5 & $<0.0001$ \\
\hline $\begin{array}{l}\text { Visual analogue scale score: } \\
\text { back pain }\end{array}$ & $7 \pm 3$ & $3 \pm 3$ & $2 \pm 3$ & $2 \pm 3$ & 2 & $<0.0001$ \\
\hline $\begin{array}{l}\text { Visual analogue scale score: } \\
\text { leg pain }\end{array}$ & $6 \pm 3$ & $2 \pm 3$ & $2 \pm 3$ & $1 \pm 3$ & 3 & $<0.0001$ \\
\hline \multicolumn{7}{|l|}{ Radiological outcomes } \\
\hline $\begin{array}{l}\text { Adjacent segment } \\
\text { degeneration }\end{array}$ & - & $1(1.8)$ & $11(19.7)$ & 22 (39.3) & - & $\begin{array}{c}0.006^{\mathrm{a})} \\
<0.0001^{\mathrm{b})} \\
0.066^{\mathrm{c}}\end{array}$ \\
\hline Grade 1 fusion & - & 22 (39.3) & 52 (92.9) & 54 (96.4) & - & $\begin{array}{c}<0.0001^{\text {a) }} \\
<0.0001^{\text {b) }} \\
0.702^{\text {c) }}\end{array}$ \\
\hline Screw loosening & - & $1(1.8)$ & $4(7.1)$ & $4(7.1)$ & - & $\begin{array}{c}0.384^{\mathrm{a})} \\
0.384^{\mathrm{b})} \\
>0.99^{\mathrm{c})}\end{array}$ \\
\hline Cage subsidence & - & $3(5.4)$ & $5(8.9)$ & $5(8.9)$ & - & $\begin{array}{c}0.763^{\text {a) }} \\
0.763^{\text {b) }} \\
>0.99^{c \mid}\end{array}$ \\
\hline
\end{tabular}

Values are presented as number (\%) or mean \pm standard deviation.

MCID, mean clinically important difference.

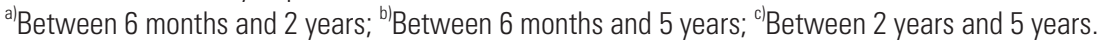

Table 5. Proportions of patients achieving MCID for clinical outcomes

\begin{tabular}{|c|c|c|c|c|}
\hline \multirow{2}{*}{ Clinical outcomes } & \multirow{2}{*}{ MCID } & \multicolumn{3}{|c|}{ Proportions of patients achieving MCID (\%) } \\
\hline & & $6 \mathrm{mo}$ & $2 \mathrm{yr}$ & $5 \mathrm{yr}$ \\
\hline Oswestry disability index & 13 & 70 & 71 & 75 \\
\hline Short-form 36 score & 5 & 66 & 82 & 82 \\
\hline Visual analogue scale score: back pain & 2 & 75 & 82 & 82 \\
\hline Visual analogue scale score: leg pain & 3 & 70 & 86 & 89 \\
\hline
\end{tabular}

MCID, mean clinically important difference.

5-year follow-ups, respectively. Subgroup analyses of patients with and without ASDeg are summarized in Table 6. No differences in demographic data or 5-year outcomes were observed between these subgroups. Fig. 2 presents an example of a patient who underwent MIS-TLIF and achieved Grade 1 fusion.

Only three patients (5.4\%) experienced clinically significant ASD during follow-up. These patients had mean ODI, NSS, SF-36, VAS-BP, and VAS-LP scores of 18, 17, 70,2 , and 1 , respectively, at 5 years postoperatively. None of these scores differed significantly from those of patients without clinical ASD. All three patients experienced symptomatic improvement with nonsurgical treatment (epidural steroid injection at adjacent levels or physiotherapy).

Notably, no major clinical complications occurred. One patient experienced the minor clinical complication of incidental durotomy, which was identified and promptly repaired intraoperatively without sequelae. Seven patients developed technical complications. Two exhibited radio- 
Table 6. Subgroup analysis of patients with and without ASDeg

\begin{tabular}{|c|c|c|c|}
\hline & Patients with ASDeg & Patients without ASDeg & $p$-value \\
\hline \multicolumn{4}{|l|}{ Demographic data } \\
\hline Total patients & 22 & 34 & - \\
\hline Females & $13(59.0)$ & $27(79.4)$ & 0.101 \\
\hline Age (yr) & $50.8 \pm 11.0$ & $56.5 \pm 11.7$ & 0.074 \\
\hline Body mass index $\left(\mathrm{kg} / \mathrm{m}^{2}\right)$ & $24.7 \pm 3.2$ & $25.2 \pm 3.5$ & 0.592 \\
\hline \multicolumn{4}{|l|}{ 5-Year outcomes } \\
\hline Oswestry disability index & $18 \pm 22$ & $14 \pm 15$ & 0.322 \\
\hline Neurogenic symptom score & $17 \pm 11$ & $10 \pm 22$ & 0.236 \\
\hline Short-form 36 score & $72 \pm 19$ & $72 \pm 21$ & $>0.99$ \\
\hline Visual analogue scale score: back pain & $2 \pm 3$ & $1 \pm 3$ & 0.228 \\
\hline Visual analogue scale score: leg pain & $1 \pm 3$ & $1 \pm 2$ & $>0.99$ \\
\hline
\end{tabular}

Values are presented as number (\%) or mean \pm standard deviation.

ASDeg, adjacent segment degeneration.
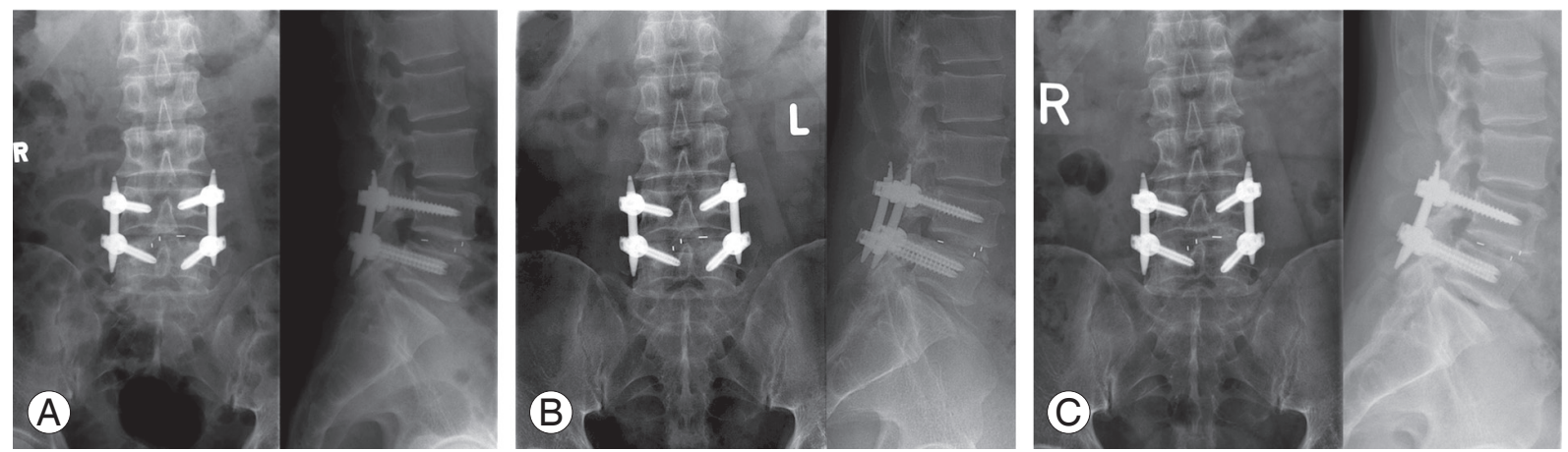

Fig. 2. Serial radiographs of a patient who underwent L4/L5 minimally invasive transforaminal lumbar interbody fusion. (A) Anteroposterior and lateral radiographs of the lumbar spine during the immediate postoperative period, showing good positioning of the screws and cage. (B) Anteroposterior and lateral radiographs of the lumbar spine at 6 months postoperatively, showing Bridwell grade II fusion. (C) Anteroposterior and lateral radiographs of the lumbar spine at 5 years postoperatively, showing Bridwell grade I fusion.

graphic evidence of screw loosening; in the first, the left inferior pedicle screw had migrated $4 \mathrm{~mm}$ posteriorly at the 2-year follow-up. However, she remained asymptomatic during follow-up and did not require surgery to remove the screw. In the second patient, radiographs obtained at 6 months showed that the right inferior pedicle screw had migrated posteriorly by up to $8 \mathrm{~mm}$. The screw was removed after the patient complained of localized pain in the corresponding area. The patient recovered well, with resolution of her pain. In one patient, evidence of S1 screw breakage was observed on a radiograph obtained at the 2-year follow-up. The patient remained asymptomatic, and no surgery was performed. Four patients had asymptomatic cage subsidence and did not require revision surgery.

\section{Discussion}

Although several methods used to achieve fusion in the lumbar spines of patients with unstable spondylolisthesis have been described [2-4], the optimal technique remains controversial. Harms and Rolinger [21] first described transforaminal interbody cage insertion in 1982. TLIF and PLIF have yielded similarly good outcomes in adults with spondylolisthesis $[22,23]$. Along with advancements in MIS techniques, TLIF has recently gained popularity as a method of achieving sagittal alignment and fusion in patients with neurogenic symptoms secondary to spondylolisthesis. MIS-TLIF, which can be performed with expandable or non-expandable working tubes and percutaneous pedicle screws, allows direct neural decompres- 
sion and avoids the muscle stripping associated with open procedures. Many authors have therefore reported good clinical and radiological outcomes for MIS-TLIF during follow-up periods of 13-34 months [24-26]. To date, only a study by Kim et al. [11] has reported 5-year clinical and radiological outcomes of patients with lumbar spondylolisthesis who underwent instrumented MIS-TLIF. In our study, we assessed both radiological and clinical outcomes according to MCID.

Our patients exhibited significant improvements in ODI, NSS, SF-36, VAS-BP, and VAS-LP at 5 years postoperatively. Kim et al. [11] reported improvements from 62 to 22 , from 6 to 4 , and from 7 to 4 in the mean ODI, VAS-BP, and VAS-LP scores, respectively, at 5 years postoperatively, in a study of 44 patients who had undergone instrumented MIS-TLIF for single-level lumbar spondyolisthesis; NSS was not an outcome evaluated. Their study is meaningful, relative to ours, for two reasons. First, both studies were conducted in Asian tertiary teaching hospitals and implemented comparable surgical techniques. However, our patients fared comparatively better. Although Kim et al. [11] did not report outcomes at 6 months and 2 years, our study demonstrated that our patients' good outcomes were sustained from 6 months to 5 years postoperatively. Furthermore, our patients' mean ODI, SF-36, VAS-BP, and VAS-LP scores met the MCID as early as 6 months postoperatively, and $75 \%-89 \%$ of patients met the MCID for each score. No previous report of MCID outcomes in a similar population is available for comparison.

The proportion of cases that achieved fusion in our study (96.4\%) is comparable to those reported in the literature [8-11]. Recently, Bevevino et al. [27] reported an overall fusion rate of $94.7 \%$, confirmed by bridging trabecular interbody bones on CT scans, in a recent systematic review. All patients in that study underwent local autologous interbody grafting of bone harvested from the pars interarticularis and facet joint of the approach side, and poly-ether-ether-ketone (PEEK) or allograft interbody cages were used in all patients. Similarly, we used PEEK cages and either autogenous posterior iliac crest bone graft or local lamina bone with DBM. We observed no relationship between the rates of fusion and those of screw loosening or cage subsidence in our patients.

The incidences of ASDeg and ASD in the current literature range from $5.2 \%$ to $100 \%$ and from $5.2 \%$ to $30.3 \%$, respectively [27-29]. Therefore, our findings corroborate those of previous studies. Cheh et al. [29] reviewed a total of 188 patients who underwent lumbar/thoracolumbar fusion with pedicle screw instrumentation for degenerative disorders. In that study, ASDeg was defined as (1) development of spondylolisthesis $>4 \mathrm{~mm}$, (2) segmental kyphosis $>10^{\circ}$, (3) complete collapse of disc space, or (4) Weiner classification worsening by more than two grades, whereas ASD was defined as (1) symptomatic spinal stenosis, (2) intractable BP, or (3) subsequent sagittal or coronal imbalance. Cheh et al. [29] observed ASDeg and ASD in 42.6\% and $30.3 \%$ patients, respectively. In our study, we used the UCLA grading scale to assess ASDeg and set a different definition of ASD. These variations might account for the lower incidences of ASDeg and ASD in our study. In their study, Cheh et al. [29] also observed worse ODI scores in patients with ASDeg relative to those without. In contrast, we did not observe this difference, a discrepancy that might be due to our smaller sample size.

Prior to advancements in MIS-TLIF, many spinal surgeons preferred PLIF technique because of anatomical familiarity. However, PLIF has been associated with moderate-to-high perioperative complication rates [30]. Scaduto et al. [30] observed that patients who underwent PLIF suffered mainly from neurologic and dura-related complications such as pseudomeningocele (16\%) and epidural hematoma (3\%). We observed no major complications in our study. Minor complications included one case of incidental durotomy as well as seven patients with technical complications of screw migration and cage subsidence. Our overall complication rate of $12.5 \%$ is comparable to those reported in the current literature $[14,29,30]$, and complications were technical and did not influence postoperative outcomes. Our study had some limitations. First, although we analyzed prospectively collected data, this was a retrospective review of a relatively small number of patients. Accordingly, we could not make meaningful statistical subgroup comparisons, especially regarding ASD. Second, outcome measures such as the time to ambulation and length of hospitalization might have been affected by differences in factors such as physiotherapists, nurses, or patient expectations. Third, no comparison group was included. Our study aimed to assess the outcomes of MIS-TLIF using the MCID criterion and to evaluate whether these outcomes were sustainable over the mid-term. Fourth, this study only included patients with neurogenic symptoms secondary to singlelevel lumbar spondylolisthesis; therefore, the results are 
not applicable to other clinical situations. On the other hand, the following steps were taken to limit bias. First, independent assessors evaluated both functional and radiological outcomes. Second, all functional outcome scores were prospectively recorded as part of our spine surgical procedure registry. Third, all patients were treated by a single surgeon and received standardized preoperative care.

\section{Conclusions}

Single-level instrumented MIS-TLIF is safe and suitable for patients with neurogenic symptoms secondary to lumbar spondylolisthesis. Clinical and radiological outcomes were sustained up to 5 years postoperatively, and many patients achieved the MCID.

\section{Conflict of Interest}

The senior author (W.M.Y.) is a consultant for Medtronic and Depuy Synthes but no funding was received for purposes of this study. All other authors have no conflicts of interests or sources of funding to disclose.

\section{Acknowledgments}

The authors thank all the staff of our institutional Orthopedic Diagnostic Centre for their efforts to maintain the rigorous Spine Registry, without which this study would have been impossible.

\section{References}

1. Herkowitz HN, Sidhu KS. Lumbar spine fusion in the treatment of degenerative conditions: current indications and recommendations. J Am Acad Orthop Surg 1995;3:123-35.

2. Cloward RB. Posterior lumbar interbody fusion updated. Clin Orthop Relat Res 1985;(193):16-9.

3. Lawton CD, Smith ZA, Barnawi A, Fessler RG. The surgical technique of minimally invasive transforaminal lumbar interbody fusion. J Neurosurg Sci 2011; 55:259-64.

4. Berjano P, Gautschi OP, Schils F, Tessitore E. Extreme lateral interbody fusion $(\mathrm{XLIF}(\mathrm{R}))$ : how I do it. Acta Neurochir (Wien) 2015;157:547-51.

5. Zhang Q, Yuan Z, Zhou M, Liu H, Xu Y, Ren Y. A comparison of posterior lumbar interbody fusion and transforaminal lumbar interbody fusion: a literature review and meta-analysis. BMC Musculoskelet Disord 2014;15:367.

6. Resnick DK, Choudhri TF, Dailey AT, et al. Guidelines for the performance of fusion procedures for degenerative disease of the lumbar spine. Part 8: lumbar fusion for disc herniation and radiculopathy. J Neurosurg Spine 2005;2:673-8.

7. Gejo R, Matsui H, Kawaguchi Y, Ishihara H, Tsuji $H$. Serial changes in trunk muscle performance after posterior lumbar surgery. Spine (Phila Pa 1976) 1999; 24:1023-8.

8. Peng CW, Yue WM, Poh SY, Yeo W, Tan SB. Clini$\mathrm{cal}$ and radiological outcomes of minimally invasive versus open transforaminal lumbar interbody fusion. Spine (Phila Pa 1976) 2009;34:1385-9.

9. Lee KH, Yue WM, Yeo W, Soeharno H, Tan SB. Clinical and radiological outcomes of open versus minimally invasive transforaminal lumbar interbody fusion. Eur Spine J 2012;21:2265-70.

10. Seng C, Siddiqui MA, Wong KP, et al. Five-year outcomes of minimally invasive versus open transforaminal lumbar interbody fusion: a matched-pair comparison study. Spine (Phila Pa 1976) 2013;38:204955.

11. Kim JS, Jung B, Lee SH. Instrumented minimally invasive spinal-transforaminal lumbar interbody fusion (MIS-TLIF): minimum 5-years follow-up with clinical and radiologic outcomes. J Spinal Disord Tech 2012 Sep 28 [Epub]. https://doi.org/10.1097/ BSD.0b013e31827415cd.

12. Fairbank JC, Pynsent PB. The Oswestry Disability Index. Spine (Phila Pa 1976) 2000;25:2940-52.

13. Daltroy LH, Cats-Baril WL, Katz JN, Fossel AH, Liang $\mathrm{MH}$. The North American spine society lumbar spine outcome assessment Instrument: reliability and validity tests. Spine (Phila Pa 1976) 1996;21:741-9.

14. Ware JE Jr, Sherbourne CD. The MOS 36-item shortform health survey (SF-36): I. Conceptual framework and item selection. Med Care 1992;30:473-83.

15. Huskisson EC. Measurement of pain. Lancet 1974;2: 1127-31.

16. Bridwell KH, Lenke LG, McEnery KW, Baldus C, Blanke K. Anterior fresh frozen structural allografts in the thoracic and lumbar spine: do they work if combined with posterior fusion and instrumentation 
in adult patients with kyphosis or anterior column defects? Spine (Phila Pa 1976) 1995;20:1410-8.

17. Ghiselli G, Wang JC, Hsu WK, Dawson EG. L5-S1 segment survivorship and clinical outcome analysis after L4-L5 isolated fusion. Spine (Phila $\mathrm{Pa} 1976$ ) 2003;28:1275-80.

18. Radcliff KE, Kepler CK, Jakoi A, et al. Adjacent segment disease in the lumbar spine following different treatment interventions. Spine J 2013;13:1339-49.

19. Copay AG, Glassman SD, Subach BR, Berven S, Schuler TC, Carreon LY. Minimum clinically important difference in lumbar spine surgery patients: a choice of methods using the Oswestry Disability Index, Medical Outcomes Study questionnaire Short Form 36, and pain scales. Spine J 2008;8:968-74.

20. Parker SL, Adogwa O, Paul AR, et al. Utility of minimum clinically important difference in assessing pain, disability, and health state after transforaminal lumbar interbody fusion for degenerative lumbar spondylolisthesis. J Neurosurg Spine 2011;14:598604.

21. Harms J, Rolinger H. A one-stager procedure in operative treatment of spondylolistheses: dorsal traction-reposition and anterior fusion (author's transl). Z Orthop Ihre Grenzgeb 1982;120:343-7.

22. Yan DL, Pei FX, Li J, Soo CL. Comparative study of PILF and TLIF treatment in adult degenerative spondylolisthesis. Eur Spine J 2008;17:1311-6.

23. Yan DL, Li J, Gao LB, Soo CL. Comparative study on two different methods of lumbar interbody fusion with pedicle screw fixation for the treatment of spondylolisthesis. Zhonghua Wai Ke Za Zhi 2008;46:497500.

24. Kim JS, Kang BU, Lee SH, et al. Mini-transforaminal lumbar interbody fusion versus anterior lumbar interbody fusion augmented by percutaneous pedicle screw fixation: a comparison of surgical outcomes in adult low-grade isthmic spondylolisthesis. J Spinal Disord Tech 2009;22:114-21.

25. Kim JS, Lee KY, Lee SH, Lee HY. Which lumbar interbody fusion technique is better in terms of level for the treatment of unstable isthmic spondylolisthesis? J Neurosurg Spine 2010;12:171-7.

26. Jhala A, Singh D, Mistry M. Minimally invasive transforaminal lumbar interbody fusion: Results of 23 consecutive cases. Indian J Orthop 2014;48:562-7.

27. Bevevino AJ, Kang DG, Lehman RA Jr, Van Blarcum GS, Wagner SC, Gwinn DE. Systematic review and meta-analysis of minimally invasive transforaminal lumbar interbody fusion rates performed without posterolateral fusion. J Clin Neurosci 2014;21:168690.

28. Bae JS, Lee SH, Kim JS, Jung B, Choi G. Adjacent segment degeneration after lumbar interbody fusion with percutaneous pedicle screw fixation for adult low-grade isthmic spondylolisthesis: minimum 3 years of follow-up. Neurosurgery 2010;67:1600-7.

29. Cheh G, Bridwell KH, Lenke LG, et al. Adjacent segment disease following lumbar/thoracolumbar fusion with pedicle screw instrumentation: a minimum 5-year follow-up. Spine (Phila Pa 1976) 2007;32:22537.

30. Scaduto AA, Gamradt SC, Yu WD, Huang J, Delamarter RB, Wang JC. Perioperative complications of threaded cylindrical lumbar interbody fusion devices: anterior versus posterior approach. J Spinal Disord Tech 2003;16:502-7. 